\title{
Examples of Extremal Lattice Fields without the Global Markov Property
}

by

\author{
Christoph KESSLER*
}

\begin{abstract}
We disprove by two examples the outstanding conjecture that every extremal Gibbs state for a given interaction on a lattice system satisfies the global Markov property.
\end{abstract}

\section{Introduction}

The global Markov property (GMP) of random fields has attracted quite a lot of attention in connection with problems of quantum field theory [8]. In the case of lattice fields, i. e. lattice spin systems, this property has been shown under different assumptions ([1], [4], [5], [2]). [5] sets up a sufficient condition for the global Markov property which is e. g. applicable in the case of maximal states for attractive interactions. By application of some heuristic argument, [5] conjectures that this condition should be trivially satisfied in the case of extremal Gibbs states which thus should be globally Markov; this conjecture has remained open. We give two examples-one with an interaction which sometimes has infinities, the other with finite interaction-where the extremal states violate the GMP.

The interaction given in these examples is far from being translation invariant. Thus the conjecture mentioned above remains open in the case of translation invariant interactions (see [6]).

Let us mention that the question of extremal Gibbs states whithout the global Markov property has been discussed also in [10]. However

Communicated by H. Araki, April 26, 1985.

* Department of Pure Mathematics, University of Hull, Hull HU6 7RX, England. 
in that paper the definition of "boundary" for a given region in a lattice differs from the usual one occuring e.g. in [5] or [6], which we also use in our text. The fact is that the Gibbs measure from [10]'s example does satisfy the GMP if the "boundary" is correctly defined.

\section{Content}

$\S 1$. Preliminaries: Statement of the global Markov property, the sufficient condition from [5], and some remarks on the example of [10].

$\S 2$. Example of an extremal Gibbs state violating the global Markov property. This example involves infinite values of the interaction.

$\S 3$. A one dimensional example of a finite interaction with nonuniqueness.

$\S 4$. Example of an extremal Gibbs state violating the global Markov property, but with finite strength of interaction. The idea of $\S 3$ will be used.

\section{§1. Preliminaries}

\subsection{Definitions, and statement of the global Markov property}

We partly adopt the notations from [5]. We work on a lattice system $\Omega=S^{\Gamma}$ where $S$ is a finite state space (in the examples it is $\{0,1\}$ ), and $\Gamma$ is some lattice (in the examples it is a subset of $\boldsymbol{Z}^{2}$, endowed with some diagonal bonds).

If $D \subseteq \Gamma$ then define $\Omega_{D}=S^{D}$; if $x$ is a configuration on $\Gamma$ (i. e., an element of $\Omega$ ) then define $x_{D}=x\left\lceil D \in \Omega_{D}\right.$.

An interaction $\Phi$ is a set $\left\{\Phi_{V} \mid V \subseteq \Gamma\right.$ finite $\}$ of mappings $\Phi_{V^{:}} \Omega_{V} \rightarrow \boldsymbol{R}$. The value $\Phi_{V}(x):=\Phi_{V}\left(x_{V}\right)$ is "the energy contributed by the part $x_{V}$ of the configuration $x " . \Phi$ is called nearest neighbour if $\Phi_{V} \equiv 0$ unless each pair $(i, j)$ in $V$ is a pair of nearest neighbours.

A probability measure $\rho$ on $(\Omega, \mathfrak{B})$ is called a Gibbs measure if it satisfies the so-called DLR-equations ([3], [7]) ( $\mathfrak{B}$ is the natural Borel algebra on $\left.\Omega=S^{\Gamma}\right)$ : For all finite $\Lambda \subseteq \Gamma, y \in \Omega_{\Lambda}, x \in \Omega$ :

$$
\rho\left(z_{\Lambda}=y \mid z_{\Gamma \backslash \Lambda}=x_{\Gamma \backslash \Lambda}\right)=\exp \left(-h_{\Lambda}^{\Phi}(\gamma, x)\right) / Z_{\Lambda, x} ;
$$

here $\rho(. \mid)$ denotes the conditional probability, $h_{\Lambda}(u, x)=\sum\left\{\Phi_{V}(u, x) \mid\right.$ 
$V \cap \Lambda \neq \emptyset\}$, and $Z_{\Lambda, x}=\sum\left\{\exp \left(-h_{\Lambda}^{\Phi}\left(y^{\prime}, x\right)\right) \mid y^{\prime} \in \Omega_{\Lambda}\right\}$.

The conditional probability introduced above depends only on the value of $x$ on $\partial \Lambda=\left\{i \mid i\right.$ is nearest neighbour of some $j \in \Lambda$, or $\exists V: \Phi_{V} \not \equiv 0$, $V \cap \Lambda \neq \emptyset$, and $i \in V\}$. This object is called the boundary of $\Lambda$ w.r.t. $\Phi$. This is the usual definition of "boundary"; $\partial \Lambda$ has to separate $\Lambda$ and $\Gamma \backslash(\Lambda \cup \partial \Lambda)$ w. r. $t$. $\Phi$.

The left hand side of (1.1) is abbreviated by $\rho\left(z_{\Lambda}=y \mid x_{\Gamma \backslash \Lambda}\right)$; it can be regarded as a probability measure $\rho_{\Lambda, x}$ on $\Omega_{\Lambda}$. Following the terminology of [9], we call $\left(\rho_{\Lambda, x}\right)_{\Lambda, x}$ the system of finite specifications for $\Phi$.

A Gibbs measure $\rho$ is called pure if it is a limit of the following form:

$$
\rho=\lim _{n \rightarrow \infty} \rho_{\Lambda_{n}, x^{0}}
$$

where $\Lambda_{n}=\{-n, \ldots, n\}^{d} \subseteq \Gamma=\mathbb{Z}^{d}$, and $x^{0}$ is some fixed configuration on $\Gamma$ (A similar definition could be done for other "well behaved" lattices $\Gamma$.).

Eq. (1.2) means that for each tame set $B$ (i. e., $B$ is measurable from within some $\Lambda_{n_{0}}$, hence it can be regarded as a subset of $\Omega_{\Lambda_{n}}$ for $n \geq n_{0}$ ),

$$
\rho(B)=\lim _{n \geqq n_{0}, n \rightarrow \infty} \rho_{\Lambda_{n}, x^{0}}(B) .
$$

A Gibbs measure is called extremal if it is not a true convex combination of different Gibbs states. Any extremal state is pure.

Finally, a given Gibbs state $\rho$ has the global Markov property iff for all $F \subseteq \Gamma$ and $f \in \mathfrak{F}_{F}$,

$$
E_{\rho}\left(f \mid \mathfrak{\Im}_{\Gamma \backslash F}\right)=E\left(f \mid \mathfrak{\Im}_{\partial F}\right),
$$

or equivalently, for all $F \subseteq \Gamma, f \in \mathfrak{F}_{F}$, and $g \in \mathfrak{F}_{G}$ :

$$
E_{\rho}\left(f \cdot g \mid \Im_{\partial F}\right)=E_{\rho}\left(f \mid \mathfrak{\Im}_{\partial F}\right) \cdot E_{\rho}\left(g \mid \mathfrak{\Im}_{\partial F}\right) \text {. }
$$

Here $G=\Gamma \backslash(F \cup \partial F)$; in general, for $D \subseteq \Gamma$, $\mathfrak{F}_{D}$ denotes the $\sigma$-algebra generated by the projection $x \mapsto x \uparrow D$. A function $f$ is in $\mathfrak{\mho}_{D}$ iff $f$ is $\widetilde{F}_{D}$-measurable and bounded.

$\rho$ has the local Markov property iff it satisfies (1.3) for all finite $F \subseteq \Gamma$.

\subsection{A sufficient condition, and the conjecture from Goldstein [5]}

This is the sufficient condition for the global Markov property 
from [5]:

(C) For all $F \subseteq \Gamma$, there is a configuration $x^{0} \in \Omega$, and a diverging sequence $\left(n_{m}\right)_{m \in N}$ such that

(i) $\quad \rho=\lim _{m \rightarrow \infty} \rho_{\Lambda_{n_{m}}, x^{0}}$,

and

(ii) $\quad \rho=\lim _{m \rightarrow \infty} \rho_{\Lambda_{n_{m}{ }^{\prime}}, x^{0}}$.

Here $\rho_{A, F, x^{0}}$ is defined by

$$
\rho_{\Lambda, F, x^{0}}(B \cap C)=\int_{C} \rho(d x) \cdot \rho_{\Lambda \cap F,\left(x_{\Lambda \backslash F}, x^{0}\right.}{ }_{\Gamma \backslash(\Lambda \backslash F)}(B)
$$

for $B \in \mathfrak{F}_{\triangle \cap F} ; C \in \mathfrak{F}_{\triangle \backslash F}$. If we now write

$$
\rho_{A, x^{0}}(B \cap C)=\int_{C} \rho_{\Lambda, x^{0}}(d x) \cdot \rho_{\Lambda \cap F,\left(x_{\Lambda \backslash F^{*}}, x^{0} \backslash \backslash(\Lambda \backslash F)\right.}(B),
$$

and compare with (1.5), then "it should be clear" that for a given Gibbs measure $\rho$ satisfying (C), (i), the measure $\rho_{\Lambda_{n_{m}}{ }^{, F}, x^{0}}$ is even closer to the limit $\rho$ than $\rho_{A_{n_{m}} \cdot x^{0}}$ is because we replace the "influence" of $\rho_{\Lambda_{n_{m}} x^{0}}$ on the part $\Lambda_{n_{m}} \backslash F$ by that of the limit measure $\rho$. Thus (C), (ii) would follow from (C), (i).

This was the heuristic argument from [5] which would imply that any pure, hence any extremal Gibbs state satisfied the GMP because it satisfies (C), (i). In the examples we present extremal Gibbs states for some interaction $\Phi$ which violate the GMP, hence (C), (ii).

\section{3. Remarks on the example from Weizsäcker [10]}

Weizsäcker [10] gives an example of a (unique hence extremal) Gibbs measure which violates the global Markov property. If we translate this example into our framework, we have a two-dimensional lattice $\boldsymbol{Z}^{2}, S=\{0,1\}$, and an interaction $\Phi=\left(\Phi_{V}\right)_{V}$ where $\Phi_{V} \not \equiv 0$ only for the following types of sets $V$ :

a) Certain three-point-sets like $\{(i, j),(i, j+1),(i+1, j)\}$; here $\Phi_{V}(x)=0$ or $\infty$ if the sum of the three $x$-values on $V$ is even or odd, respectively. 
b) Certain two-point-sets like $\{(i, j),(i+1, j)\}$, i. e., $V$ contains a couple of nearest neighbours. Here $\Phi_{V}(x)=0$ or $\infty$ if the two $x$ values are equal or not equal, respectively.

c) The two-point-set $V_{0}=\{(-1,0),(1,0)\}$ which links two nonneighbouring sites. $\Phi_{V_{0}}$ is as in b).

The boundary $\partial \Lambda$ now is defined only in terms of nearest neighbours $(\partial \Lambda=\{i \notin \Lambda \mid \exists j \in \Lambda$ such that $(i, j)$ are nearest neighbours $\})$. Now, if $F$ is the right half-plane, $\partial F$ is only the $y$-axis; so $\Phi_{V_{0}} \operatorname{links} F$ and $\Gamma \backslash(F \cup \partial F)$. Thus it is not surprising that-with this definition of $\partial F$ and $\mathscr{F}_{\partial F}$-the GMP is violated for the (unique) Gibbs state $\rho$ for $\Phi$. The real "wonder" is that the local Markov property is satisfied if we admit diagonal bonds; this depends heavily on the choice of the sets $V$ supporting $\Phi$.

As mentioned above, $\rho$ has the global Markov property if $\partial F$ is defined as in 1.1 .

\section{§2. Counterexample to the Global Markov Property}

This counterexample involves infinite energies. Choose $\Gamma=\{(-1, i)$, $(0, i),(1, i) \mid i \in N\}$. This is a strip of width 3 along the positive $y$ axis.

$\Phi$ is supported by the sets $V_{i}=\{(0, i),(-1, i),(-1, i+1)\}$ and $V^{i}=\{(0, i),(1, i),(1, i+1)\} . \quad \Phi_{V^{i}}(x)$ has the value $\infty$ or 0 if the sum of the three $x$-values is $0 \bmod 2$ or $1 \bmod 2$, respectively. Similarly for $\Phi_{V_{i}}$.

$\Phi$ can be viewed as nearest neighbour if we admit some diagonal bonds.

Now, for $n \in N$, let $\Lambda_{n}=\{(-1, i),(0, i),(1, i) \mid 0 \leqq i \leqq n\} ; \partial \Lambda_{n}$ then is $\{(-1, n+1),(0, n+1),(1, n+1)\}$.

We abbreviate the finite specifications $\rho_{\Lambda_{n}, x}$ by $\rho_{n, x}$ and look upon $x \in \Omega_{\partial \Lambda_{n}}$ as of a triple $\left(x_{-1}, x_{0}, x_{1}\right)$ with components from $\{0,1\}$. If necessary we specify such an $x$ by " 111 " etc.

By the definition of $\Phi$, we now have for $m \leqq n-2$, and each set $B$ which is measurable from within $\Lambda_{m}$ (thus $B$ can be seen as a subset of $\Omega_{\Lambda_{m}}$ as well as of $\Omega_{\Lambda_{n}}$ ): 


$$
\begin{array}{ll}
\rho_{n, x}(B)=(1 / 4) \cdot \sum\left\{\rho_{m, y}(B) \mid y_{-1}=y_{1}\right\} & \text { if } x_{-1}=x_{1} ; \\
\rho_{n, x}(B)=(1 / 4) \cdot \sum\left\{\rho_{m, y}(B) \mid y_{-1} \neq y_{1}\right\} & \text { if } x_{-1} \neq x_{1} .
\end{array}
$$

In the first case $x$ will be called good, in the second case bad; the same terms will be applied for configurations $x_{m}=\left(x_{-1, m}, x_{0, m}, x_{1, m}\right)$.

Since the two probabilities differ in general (example : for $n \geqq 1$ we have $\rho_{n, 111}\left(z_{0}\right.$ is good $)=1 ; \rho_{n, 001}\left(z_{0}\right.$ is good $\left.)=0\right)$, the following condition for the existence of the thermodynamic limit $\lim _{n \rightarrow \infty} \rho_{n, x_{n}}$ holds:

$$
\begin{array}{r}
\lim _{n \rightarrow \infty} \rho_{n, x_{n}} \text { exists iff } x_{n} \text { is good for almost all } n \in N, \\
\text { or } x_{n} \text { is bad for almost all } n \in N .
\end{array}
$$

The two limits will be called $\rho_{\text {good }}$ and $\rho_{b a d}$; from the above we conclude $\rho_{\text {good }} \neq \rho_{b a d} . \rho_{\text {good }}$ and $\rho_{b a d}$ are the only pure, hence the only extremal Gibbs states for our system.

Now, for a contradiction to the global Markov property, let $F=$ $\{(1, i) \mid i \geqq 0\}$, thus $\partial F=\{(0, i) \mid i \geqq 0\}$, and $f=I_{\left\{z_{10}=1\right\}}, g=I_{\left\{z_{-10}=1\right\}}$. (I. is the indicator function).

\section{Proposition 2.1.}

a) $E_{\rho}\left(h \mid \mathscr{F}_{\partial F}\right)=1 / 2$ a.s. for $\rho=\rho_{\text {good }}$ or $\rho_{\text {bad }}, h=f$ or $g$.

b) $E_{\rho_{\text {good }}}\left(f \cdot g \mid \mathscr{F}_{\partial F}\right)=1 / 2$ a.s., $E_{\rho_{\text {bad }}}\left(f \cdot g \mid \mathscr{F}_{\partial F}\right)=0$ a.s.

Thus the GMP is not valid since in b) we had to expect the value $1 / 4$ a.s.

Proof. Call $\nu \in S^{n+1}$ even if $\#\left\{i \mid \nu_{i}=0\right\}$ is even; otherwise $\nu$ is called odd. For given $n \in N$, $\nu \in S^{n+1}$, and some event $B$ we abbreviate $\rho\left(B \mid\left(z_{00} \ldots z_{0 n}\right)=\nu\right)$ by $\rho(B \mid \nu)$; here $\rho$ indicates $\rho_{\text {good, }} \rho_{b a d}$, or some $\rho_{k, x}$ where $k$ is so large that $B$ is measurable from within $\Lambda_{k}$.

Ad a) E. g., let $\rho=\rho_{\text {good, }}$ and $h=f$. Then we have to show, for $n \geqq 2$ and $\nu \in S^{n+1}$,

$$
\rho_{\text {good }}\left(z_{10}=1 \mid \nu\right)=1 / 2
$$

Now, in general, for $x \in \Omega_{\partial \Lambda_{n}}$

$$
\rho_{\text {good }}\left(z_{n+1}=x \mid \nu\right)=\rho_{\text {good }}\left(z_{n+1}=x\right),
$$

because the left hand side is independent of $\nu$, see the definition of $\Phi$. Thus 


$$
\begin{aligned}
\rho_{\text {good }}\left(z_{10}=1 \mid \nu\right) & =\sum\left\{\rho_{\text {good }}\left(z_{n+1}=x \mid \nu\right) \cdot \rho_{n, x}\left(z_{10}=1 \mid \nu\right) \mid x \in \Omega_{\partial \Lambda_{n}}\right\} \\
& =\sum\left\{\rho_{\text {good }}\left(z_{n+1}=x\right) \cdot \rho_{n, x}\left(z_{10}=1 \mid \nu\right) \mid x \in \Omega_{\partial \Lambda_{n}}\right\}
\end{aligned}
$$

But the first factor is $1 / 4$ for exactly the good $x$, and 0 for the bad $x$. The second factor is 1 iff $\nu$ is even and $x=\ldots 1$, or $\nu$ is odd and $x=\ldots 0$; otherwise this factor is 0 . (A dot means an arbitrary value.) In any case it is 1 for exactly two of the four good $x$-values. Thus

$$
\rho_{\text {good }}\left(z_{10}=1 \mid \nu\right)=2 / 4=1 / 2 .
$$

$A d b$ ) We prove the first formula; the second one can be shown similarly. We have to show, for each $n \geqq 2$, and $\nu \in S^{n+1}$,

$$
\rho_{\text {good }}\left(z_{10}=1=z_{-10} \mid \nu\right)=1 / 2 \text {. }
$$

Now, as in the proof of a) (see (2.4), (2.5)), and by the local Markov property,

$$
\rho_{\text {good }}\left(z_{10}=1=z_{-10} \mid \nu\right)=\sum\left\{\rho_{n, x}\left(z_{10}=1 \mid \nu\right) \cdot \rho_{n, x}\left(z_{-10}=1 \mid \nu\right) \mid x \text { good }\right\} .
$$

But the product is 1 if $x=1.1$ and $\nu$ is even, or $x=0.0$ and $\nu$ is odd. Otherwise the product is zero. In any case it is 1 for exactly two of the good $x$; hence

$$
\rho_{\text {good }}\left(z_{10}=1=z_{-10} \mid \nu\right)=2 / 4=1 / 2 .
$$

If you want to avoid infinite energies, i. e., infinite values of $\Phi$, you are referred to $\S \S 3$ and 4 . There we replace the infinities by very fastly increasing finite values which have-for our problem of the GMPthe same effect.

\section{§3. A One Dimensional Example}

The following nearest neighbour interaction $\Phi^{\prime}$ on the lattice system $\Omega^{\prime}=S^{\Gamma^{\prime}}, S=\{0,1\}, \Gamma^{\prime}=\boldsymbol{N}$, is an attractive interaction with finite values which has two different extremal Gibbs states.

Define $\Phi_{V}^{\prime} \equiv 0$ unless $V=V_{i}^{\prime}=\{i, i+1\}$ for $i \in \mathbb{N}$. In this case

$$
\Phi_{V_{1}^{\prime}}^{\prime}\left(x_{i}, x_{i+1}\right)=0 \text { or } b_{i}>0 \text { if } x_{i}=x_{i+1} \text { or } x_{i} \neq x_{i+1} \text {. }
$$

Remark. If we put $\Phi_{V_{1}^{\prime}}^{\prime}\left(x_{i}, x_{i+1}\right)=0$ or $\infty$ if $x_{i}=$ or $\neq x_{i+1}$, then it is clear that there are exactly two different extremal Gibbs measures. 
If we now choose large enough $b_{i}$ (the choice will be made later), then we will obtain the same effect.

Proposition 3. 1. Let $\varepsilon \in(0,1 / 2)$ be given. Then there exists a monotonically increasing sequence $\left(b_{i}\right)_{i \in N}$ such that for all $n>m \geqq 0$

$$
p_{m n}^{\prime}:=\rho_{n, 1}^{\prime}\left(z_{m}=0\right)<\varepsilon .
$$

Here $\rho_{n, 1}^{\prime}$ is the finite specification determined by the value 1 on the only site $n+1$ on $\partial \Lambda_{n}$.

\section{Conclusions.}

1) Since always, by the local Markov property,

$$
\rho_{n, x}^{\prime}\left(y \uparrow \Lambda_{m}=z \uparrow \Lambda_{m}\right)=a \cdot \rho_{n, x}^{\prime}\left(y_{m}=z_{m}\right),
$$

where $a$ is a factor independent of $n$, and since $\varepsilon<1 / 2$, we conclude

$$
\begin{array}{r}
\lim _{n \rightarrow \infty} \rho_{n, x_{n}}^{\prime} \text { exists iff } x_{n}=1 \text { for almost all } n \in N \\
\text { or } x_{n}=0 \text { for almost all } n \in N .
\end{array}
$$

The limits will be denoted by $\rho_{1}^{\prime}$ and $\rho_{0}^{\prime}$, respectively; the two limit measures differ.

2) It is clear, since $b_{i}>0$, that $\Phi^{\prime}$ is attractive; thus $\rho_{1}^{\prime}$ is maximal, and $\rho_{0}^{\prime}$ is minimal. By (3.4), there are only two pure, hence only two extremal Gibbs states. So $\rho_{0}^{\prime}$ and $\rho_{1}^{\prime}$ are the only two extremal Gibbs states.

3) For all $m \in N$, and $x, y \in S$ :

$$
\begin{aligned}
& \rho_{x}^{\prime}\left(z_{m}=y\right)=\lim _{n \rightarrow \infty} p_{m n}^{\prime}=: p_{m}^{\prime}<\varepsilon \text { if } x \neq y ; \\
& \rho_{x}^{\prime}\left(z_{m}=y\right)=\lim _{n \rightarrow \infty} q_{m n}^{\prime}=q_{m}^{\prime}>(1-\varepsilon) \text { if } x=y .
\end{aligned}
$$

Here $q_{m n}^{\prime}=\left(1-p_{m n}^{\prime}\right)$, and $q_{m}^{\prime}=\left(1-p_{m}^{\prime}\right)$.

Proof of proposition 3.1. Let $m<n$ be given, and define

$$
Z_{m, n, 1}=\sum\left\{\exp \left(-h_{m, n, 1}(\nu)\right) \mid \nu \in S^{(m, \ldots, n)}\right\} \geqq 1,
$$

where, for $\nu \in S^{[m, \ldots, n\}}$, and $k(\nu)=\max \left\{i \mid \nu_{i}=0\right\}$,

$$
h_{m, n, 1}(\nu)=\sum\left\{b_{i} \mid i=m, \ldots, n, \nu_{i}=\nu_{i+1}\right\} \geqq k_{k}(\nu) .
$$

Then

$$
\begin{aligned}
p_{m n}^{\prime} & =\rho_{n, 1}^{\prime}\left(Z_{m}=0\right) \\
& =\sum\left\{\exp \left(-h_{m, n, 1}(\nu)\right) / Z_{m, n, 1} \mid \nu \in S^{(m, \cdots, n)}, \nu_{m}=0\right\}
\end{aligned}
$$




$$
\begin{aligned}
& \leqq e^{-b_{m}}+\sum\left(\#\left\{\nu_{m}=0=\nu_{k}, \nu_{k+1}=\ldots=\nu_{n+1}=1\right\} \cdot e^{-b_{k}} \mid m<k \leqq n\right\} \\
& =e^{-b_{m}}+\sum\left\{2^{i-1} e^{-b_{i}+m} \mid i=1, \ldots, k-m\right\} \\
& \leqq e^{-b_{0}}+\sum_{i \in N} 2^{i-1} e^{-b_{i}} \\
& <\varepsilon
\end{aligned}
$$

where the last two inequalities hold by an appropriate choice of a monotonically increasing sequence $\left(b_{i}\right)_{i}$ uniformly in $n$ and $m$.

\section{§4. Counterexample to the Global Markov Property}

The situation is nearly the same as in example 2 (recall the definition of $\Gamma, V_{i}, V^{i}, \Lambda_{n}$, and $\partial \Lambda_{n}$ ), but we define the interaction $\Phi$ as follows: $\Phi_{V} \equiv 0$ unless $V=V^{i}$ or $V^{i}$ for some $i \in \mathbb{N} ; \Phi_{V_{i}}(x)=d_{i}$ or 0 if the sum of the three $x$-values on $V_{i}$ is $0 \bmod 2$ or $1 \bmod 2$. Similarly we define $\Phi_{V^{i}}$

Now we choose the sequence $\left(d_{i}\right)_{i \in N}$ : Let $\varepsilon<1 / 6$ be given, and let $\left(b_{i}\right)_{i \in N}$ be a sequence from example 3 for this $\varepsilon$ (see the proof of (3.1)). Then put

$$
d_{i}=\operatorname{arcosh}\left(\exp \left(b_{i}\right)\right)>0 \quad\left(\text { thus } b_{i}=\ln \left(\cosh \left(d_{i}\right)\right)\right)
$$

These $d_{i}$ will work.

Proposition 4.1. If $n>m$, then, with $p_{m n}^{\prime}$ and $q_{m n}^{\prime}$ from (3.2) and (3.5),

$$
\begin{gathered}
\rho_{n, x}\left(z_{m}=y\right)=\rho_{n, 111}\left(z_{m} \text { is good }\right) / 4=q_{m n}^{\prime} / 4>(1-\varepsilon) / 4 \\
\text { if }(x \text { is good iff } y \text { is good }) \\
\rho_{n, x}\left(z_{m}=y\right)=\rho_{n, 111}\left(z_{m} \text { is bad }\right) / 4=p_{m n}^{\prime} / 4>\varepsilon / 4 \\
\text { if }(x \text { is good iff } y \text { is bad }) .
\end{gathered}
$$

Proof. First we compute some probabilities: For $k \in \mathbb{N}$, define $Z_{k}=2+4 \cdot e^{-d_{k}}+2 \cdot e^{-2 d_{k}}=2 \cdot\left(1+e^{-d_{k}}\right)^{2}$. Then

$$
\rho_{k, x}\left(z_{k}=y\right)=\quad \text { a) } 1 / Z_{k}, \quad \text { b) } e^{-d_{k}} / Z_{k}, \quad \text { c) } e^{-2 d_{k}} / Z_{k},
$$

in the case of a) $y_{1}+y_{0}+x_{1}=1 \bmod 2, l= \pm 1$ (these are exactly two $y$ of the same "sort" as $x$, b) $y$ is good iff $x$ is good, and c) $y_{1}+y_{0}+x_{1}=0 \bmod 2, l= \pm 1$ (these are the other two $y$ of the same 
"sort" as $x$ ). We prove the assertion by induction on $j=n-m$.

Case $j=1$ :

Let $x, y$ be good (the other cases are similar), and apply (4.3) for $k=m$ and $k=m+1$ in order to obtain

$$
\begin{aligned}
\rho_{m+1, x}\left(z_{m}=y\right) & =\sum\left\{\rho_{m+1, x}\left(z_{m+1}=u\right) \cdot \rho_{m, u}\left(z_{m}=y\right) \mid u=000 \ldots 111\right\} \\
& =\left[\left(1+e^{-2 d_{m}}\right)\left(1+e^{-2 d_{m+1}}\right)+4 \cdot e^{-d_{m}-_{m+1}}\right] / Z_{m} \cdot Z_{m+1} \\
& \stackrel{(+)}{=}\left(1+e^{-b_{m}-b_{m+1}}\right) / 4\left(1+e^{-b_{m}}\right)\left(1+e^{-b_{m+1}}\right) \\
& =q_{m m+1}^{\prime} / 4 .
\end{aligned}
$$

Step $j \longrightarrow j+1:$

Let $n=m+j$, and $x, y$ be good (the other cases are similar). Assume the assertion for this $j$ and all possible $\tilde{x}, \tilde{y}$ (induction hypothesis). Then

$$
\begin{aligned}
\rho_{n+1, x}\left(z_{m}=y\right) & =\sum\left\{\rho_{n+1, x}\left(z_{m+1}=u\right) \cdot \rho_{m, u}\left(z_{m}=y\right) \mid u=000 \ldots 11\right\} \\
& \stackrel{(*)}{=}\left[2\left(1+e^{-2 d_{m}}\right) \cdot q_{m+1 n+1}^{\prime}+4 \cdot e^{-d_{m}} \cdot p_{m+1 n+1}^{\prime}\right] / 4 \cdot Z_{m} \\
& \stackrel{(+)}{=} q_{m+1 n+1}^{\prime} / 4\left(1+e^{-b_{m}}\right)+p_{m+1 n+1}^{\prime} e^{-b_{m}} / 4\left(1+e^{-b_{m}}\right) \\
& =q_{m n+1}^{\prime} / 4 .
\end{aligned}
$$

At $\left(^{*}\right)$, we used (4.3) and the induction hypothesis, at $(+)$ the definition of the $d_{i}$.

\section{Proposition 4.2.}

$$
\begin{array}{r}
\lim _{n \rightarrow \infty} \rho_{n, x_{n}} \text { exists iff } x_{n} \text { is good for almost all } n \in N \text { or } \\
x_{n} \text { is bad for almost all } n \in N .
\end{array}
$$

The two limit measures, denoted by $\rho_{\text {good }}$ and $\rho_{b a d}$, are different and the only extremal Gibbs states.

Proof. Let $B$ be a tame set of the form $B=\left\{z \uparrow \Lambda_{m+1}=y\right\}$ for some $y \in \Omega_{\Lambda_{m+1}}$; let $u=y_{m+1}$. Then, if $n>m+1$, we have by the local Markov property,

$$
\begin{aligned}
\rho_{n, x}(B) & =\rho_{n, x}\left(z_{m+1}=u\right) \cdot \rho_{m, u}\left(z \nmid \Lambda_{m}=y \uparrow \Lambda_{m}\right) \\
& =(1 / 4) r_{m+1 n}^{\prime} \rho_{m, u}\left(z \uparrow \Lambda_{m}=y \uparrow \Lambda_{m}\right),
\end{aligned}
$$

where $r_{m+1 n}^{\prime}=p_{m+1 n}^{\prime}$ if ( $x$ good iff $u$ bad); otherwise $r_{m+1 n}^{\prime}=q_{m+1 n}^{\prime}$. 
But since $\varepsilon<1 / 6,\left|q_{m+1 n}^{\prime}-p_{m+1 n}^{\prime}\right|>2 / 3>0$ uniformly in $n \in \mathbb{N}$, so $\lim _{n \rightarrow \infty} \rho_{n, x_{n}}(B)$ exists iff $x_{n}$ is good for almost all $n \in \mathbb{N}$ or $x_{n}$ is bad for almost all $n \in \mathbb{N}$. The proposition follows from this since each tame set can be constructed from finitely many sets as above.

Proposition 4.3. $\rho_{\text {good }}$ and $\rho_{b a d}$ violate the global Markov property.

Proof. (E. g. for $\left.\rho_{\text {good }}\right)$ Let $F=\{(1, i) \mid i \in N\}, \quad \partial F=\{(0, i) \mid i \in \mathbb{N}\}$, and $f=I_{\left(z_{10}=1\right\}}, g=I_{\left[z_{-10}=1\right\}}$, as in example 2. We show

$$
\begin{aligned}
& E_{\rho_{g o o d}}\left(f \mid \mathscr{F}_{\partial F}\right)=E_{\rho_{\text {good }}}\left(g \mid \mathscr{F}_{\partial F}\right)=1 / 2 \text { a. s., } \\
& E_{\rho_{\text {good }}}\left(f \circ g \mid \mathscr{F}_{\partial F}\right) \geqq(1 / 2) \cdot(1-3 \varepsilon) \text { a. s. }
\end{aligned}
$$

This is enough since for $\varepsilon<1 / 6$ the last value never reaches $1 / 4$ which would be expected under the GMP.

First, let $\rho^{\prime \prime}$ be the one dimensional measure from example 3 , but the $b_{i}$ replaced by the $d_{i}$. Similarly define $p_{m n}^{\prime \prime}$ and $q_{m n}^{\prime \prime}$, e. g., $p_{m n}^{\prime \prime}=\rho_{n, 1}^{\prime \prime}\left(z_{m}=0\right)$. Since - by $(4.1)-\exp \left(-d_{i}\right) \leqq \exp \left(-b_{i}\right)$, we get

$$
p_{m n}^{\prime \prime} \leqq p_{m n}^{\prime}<\varepsilon, \quad q_{m n}^{\prime \prime} \geqq q_{m n}^{\prime}>(1-\varepsilon) .
$$

For $\nu \in S^{n+1}$ we already know the definition of "even" and "odd", and the notation $\rho(B \mid \nu)$, see the proof of proposition 2.1. We compute easily

$$
\begin{aligned}
& \rho_{n, x}\left(z_{10}=1 \mid \nu\right)=\left\{\begin{array}{l}
q_{0 n}^{\prime \prime} \text { if } x_{1}=1, \nu \text { even, or } x_{1}=0, \nu \text { odd, } \\
p_{0 n}^{\prime \prime} \text { otherwise, }
\end{array}\right. \\
& \rho_{n, x}\left(z_{-10}=1 \mid \nu\right)=\left\{\begin{array}{l}
q_{0 n}^{\prime \prime} \text { if } x_{-1}=1, \nu \text { even, or } x_{-1}=0, \nu \text { odd, } \\
p_{0 n}^{\prime \prime} \text { otherwise. }
\end{array}\right.
\end{aligned}
$$

Now, by the local Markov property, we get from (4.8) and (4.9):

$$
\rho_{n, x}\left(z_{10}=1=z_{-10} \mid \nu\right)=\left\{\begin{array}{l}
p_{0 n}^{\prime \prime 2} \text { if } x=0.0, \nu \text { even or } x=1.1, \nu \text { odd, } \\
p_{0 n}^{\prime \prime} \cdot q_{0 n}^{\prime \prime} \text { if } x \text { is bad } \\
q_{0 n}^{\prime \prime 2} \text { if } x=1.1, \nu \text { even or } x=0.0, \nu \text { odd }
\end{array}\right.
$$

(a "." means arbitrary values).

For a proof of (4.5) and (4.6), let $n \in \mathbb{N}$ and $\nu \in S^{n+1}$ be given. Then

$$
\rho_{g o o d}\left(z_{10}=1 \mid \nu\right)=\sum\left\{\rho_{g o o d}\left(z_{n+1}=x \mid \nu\right) \cdot \rho_{n, x}\left(z_{10}=1 \mid \nu\right) \mid x \in \Omega_{\partial \Lambda_{n}}\right\}
$$




$$
\begin{aligned}
& \stackrel{(+)}{=} \sum\left\{\rho_{\text {good }}\left(z_{n+1}=x\right) \cdot \rho_{n, x}\left(z_{10}=1 \mid \nu\right) \mid x \in \Omega_{\partial \Lambda_{n}}\right\} \\
& =(2 / 4) \cdot\left(\rho_{\text {good }}\left(z_{n+1} \text { good }\right)+\rho_{\text {good }}\left(z_{n+1} b a d\right) \cdot\left(p_{0 n}^{\prime \prime}+q_{0 n}^{\prime \prime}\right)\right. \\
& =1 / 2
\end{aligned}
$$

and

$$
\begin{aligned}
\rho_{\text {good }}\left(z_{10}=1=z_{-10} \mid \nu\right) \stackrel{(+)}{=} & \sum\left\{\rho_{\text {good }}\left(z_{n+1}=x\right) \cdot \rho_{n, x}\left(z_{10}=1=z_{-10} \mid \nu\right) \mid x \in \Omega_{\partial \Lambda_{n}}\right\} \\
= & (2 / 4) \cdot \rho_{\text {good }}\left(z_{n+1} \text { good }\right) \cdot\left(p_{0 n}^{\prime \prime 2}+q_{0 n}^{\prime \prime 2}\right) \\
& +(4 / 4) \cdot \rho_{\text {good }}\left(z_{n+1} \text { bad }\right) \\
\geqq & (1 / 2)(1-\varepsilon)(1-\varepsilon)^{2} \\
\geqq & (1 / 2)(1-3 \varepsilon) .
\end{aligned}
$$

(4.5) and (4.6) are valid since $n$ is arbitrary, and $\mathscr{F}_{\partial F}$ is generated by the $\mathscr{F}_{\partial F}$-measurable tame sets. At $(+)$ we used the identity $\rho_{\text {good }}\left(z_{n+1}=x \mid \nu\right)=\rho_{\text {good }}\left(z_{n+1}=x\right)$ which follows from the definition of $\Phi$.

\section{References}

[ 1 ] Albeverio, S., Høegh-Krohn, R. and Olsen, C., The global Markov property for lattice systems. J. Multiv. Anal. 11 (1981), 599-607.

[2] Bellissard, J., and Høegh-Krohn, R., Compactness and the maxımal Gibbs state for random Gibbs fields on a lattice. Comm. Math. Phys. 84 (1982), 297-327.

[3] Dobrushin, R. L., Gibbsian random fields for lattice systems with pairwise interactions. Funct. Anal. Appl. 2 (1968), 292-301.

[4] Föllmer, H., On the global Markov property. In : Quantum Fields - Algebras, Processes (L. Streit, Ed.), Springer Wien, 1980, 293-301.

[5] Goldstein, S., Remarks on the global Markov property. Comm. Math. Phys. 74 (1980), 194-215.

[6] Higuchi, Y., A remark on the global Markov property for the $d$-dimensional Ising model. Proc. Japan Acad., 60, Ser. A (1984), 49-52.

[7] Lanford III, O.E. and Ruelle, D., Observables at infinity and states with short range correlations in statistical mechanics. Comm. Math. Phys. 13 (1969), 194-215.

[8] Nelson, E., Construction of quantum fields from Markov fields. J. Funct. Anal. 12 (1973), 97-112.

[9] Preston, C., Random Fields. Lecture Notes in Mathematics 534, Springer Berlin 1976

[10] Von Weizsäcker, H., A simple example concerning the global Markov property of lattice random fields. $8^{\text {th }}$ Winter School on Abstract Analysis, Math. Inst. of the Czech Acad. of Sciences, Praha, 1980. 